\title{
Lipid is absorbed in the stomach by epithelial neoplasms (adenomas and early cancers): a novel functional endoscopy technique
}

Authors

Institutions
Kensei Ohtsu' ${ }^{1}$, Kenshi Yao ${ }^{2}$, Kazuhisa Matsunaga ${ }^{3}$, Takashi Nagahama' ${ }^{1}$, Takao Kanemitsu' ${ }^{1}$, Yu Matsushima', Motochika Yasaka ${ }^{1}$, Yoichiro Ono ${ }^{1}$, Shoko Fujiwara ${ }^{1}$, Takashi Hisabe ${ }^{1}$, Yasuhiro Takaki ${ }^{1}$, Fumihito Hirai ${ }^{1}$, Toshiyuki Matsui ${ }^{1}$, Teruyo Hanada ${ }^{4}$, Kentaro Imamura ${ }^{5}$, Hiroshi Tanabe ${ }^{5}$, Akinori Iwashita ${ }^{5}$, Toshio Shimokawa ${ }^{6}$

Institutions are listed at the end of article. submitted

8. December 2014 accepted after revision 16. March 2015

\section{Bibliography}

DOI http://dx.doi.org/

10.1055/s-0034-1392095

Published online: 12.6.2015

Endosc Int Open 2015; 03:

E318-E322

(c) Georg Thieme Verlag KG

Stuttgart · New York

E-ISSN 2196-9736

\section{Corresponding author}

Kensei Ohtsu, MD

Department of

Gastroenterology

Fukuoka University Chikushi

Hospital

1-1-1 Zokumyoin

Chikushino

Fukuoka 818-8052

Japan

Fax: +81-(0)92-929-2630

k.ohtsu0120@gmail.com

\section{License terms}

(ब) (1) $\Theta \circledast$
Background and study aims: The authors have successfully demonstrated that the white opaque substance (WOS) identified in gastric epithelial neoplasms is an accumulation of minute lipid droplets on the epithelial neoplasm. It is not known whether the lipid droplets originate from externally ingested lipids (typically foods). The purpose of this study was to investigate whether the oral ingestion of foods containing emulsified fats increases the density of the WOS in epithelial neoplasms.

Patients and methods: We examined 92 gastric epithelial neoplastic lesions in 89 patients. The patients were given emulsified fatty foods before the procedure, and magnifying endoscopy with narrow-band imaging (M-NBI) was used to image the lesions. An increase in WOS density after the ingestion of emulsified fatty foods was defined as a positive fat-loading test result.

The patients were divided into the following

\section{Introduction}

\section{$\nabla$}

A number of studies have investigated the utility of diagnostic systems for gastric epithelial neoplasms based on the identification of subepithelial microvascular architecture and microsurface architecture through magnifying observation. The validity and legitimacy of these reports are widely acknowledged [ $1-10]$. Through the examination of gastric epithelial neoplasia and precancerous intestinal metaplasia with magnifying endoscopy, the authors detected the presence of a white substance that is not permeable to light, which we called "white opaque substance" (WOS) in our earlier report [10]. We found that the morphologic characteristics of the WOS can be used to distinguish between cancerous and noncancerous lesions [11]. More recently, we demonstrated that the WOS is an accumulation of tiny lipid droplets in the epithelium $[12,13]$. groups: control group, no emulsified fat administered; group 1, fatty food administered 16 hours prior; group 3, fatty food administered both 16 and 4 hours prior. The proportion of positive fat-loading test results was determined in all groups.

Results: The rates of positive fat-loading test results were as follows: control group, $9 \%$; group $1,26 \%$; group $2,52 \%$; group $3,78 \%$. The increase in the rates of positive fat-loading test results in groups 2 and 3 relative to the rate in the control group was statistically significant (chi-squared test).

Conclusions: This study demonstrated for the first time that the ingestion of external lipids causes lipid droplets to aggregate in situ on the gastric epithelial neoplasm. These results can be used to develop a novel functional endoscopy technique that harnesses the lipid absorption capacity of neoplasms.

However, we do not yet know the origin or source of the lipid droplets.

In this study, we administered an oral lipid preparation to patients with gastric epithelial neoplasia and determined endoscopically whether this was associated with an increase in lipid droplets in the epithelial neoplasms.

\section{Materials and methods}

\section{$\nabla$}

\section{Study design and participants}

The study was a single-center, nonrandomized, unblinded, prospective intervention study, performed in accordance with the provisions of the Helsinki Declaration. The study design was approved by the University of Fukuoka Clinical Trials Review Board. All participating patients were supplied with an explanation of the study and provided written consent. 
We enrolled consecutive patients with gastric epithelial neoplasia (adenoma or early gastric cancer) who underwent endoscopic mucosal resection (EMR) or endoscopic submucosal dissection (ESD) at Fukuoka University Chikushi Hospital between January 2011 and November 2012, and who satisfied the following inclusion criteria:

- Magnifying endoscopy with narrow-band imaging (M-NBI) before ESD of the gastric epithelial neoplasm yielded at least three in-focus images on the oral side of the target lesion.

- The patient had an Eastern Cooperative Oncology Group (ECOG) performance status of either 0 or 1 [14].

- The patient was 20 years of age or older at the time of registration.

- The patient was capable of ingesting the test meal.

- The patient had provided written consent to participate in the study.

Patients were excluded from the study based on the following criteria: lesions with associated inflammation, ulceration, or hemorrhage that restricted observation of the lesion surface; history of gastrectomy; serious concomitant disease or illness; lactose intolerance.

\section{Histopathologic diagnosis}

Histopathologic diagnosis of the lesions targeted in the fat-loading test was made through the examination of ESD specimens, based on the Vienna classification system $[15,16]$ :

- C1, negative for neoplasia

- C2, indefinite for neoplasia

- C3, mucosal low grade neoplasia (low grade dysplasia/adenoma)

- C4, mucosal high grade neoplasia

- C4.1, high grade dysplasia/adenoma

- C4.2, noninvasive carcinoma (carcinoma in situ)

- C4.3, intramucosal carcinoma

- C5, submucosal invasion by tumor

C3 lesions were classified as low grade adenomas. C4 and C5 lesions were classified as early gastric cancers and further subdivided into differentiated and undifferentiated types.

\section{Endoscopy system and endoscopy procedures}

We used Evis Lucera Spectrum, an electronic NBI endoscopy system (Olympus, Tokyo, Japan) that features the GIF-Q240Z upper gastrointestinal tract endoscope with optical magnification (Olympus) and has a maximum resolution of $7.9 \mu \mathrm{m}$ [2]. A soft black hood attachment (MAJ-1989, Olympus [4]) was fitted to the tip of the endoscope during examination. Structural enhancement was set to level B8 for NBI observation, and NBI color mode 1 was used. The examination technique was as reported previously [4].

For the purpose of this study, we examined the oral end of the lesion. After initial examination with conventional white-light imaging, we examined the lesion with M-NBI and took photographs of three different sites at maximum magnification. Still images were stored in the Solemio ENDO version 3.3 image recording system (Olympus). Endoscopic examinations were performed by specialists accredited by the Japan Gastroenterological Endoscopy Society or with similar qualifications.

\section{Administration of emulsified fatty food}

The patients were given ice cream, which contains both fats and emulsifiers. We used Sou brand (Lotte Co., Ltd., Tokyo, Japan), which had a fat content of $12 \%$ and $241 \mathrm{kcal}$ per $190-\mathrm{mL}$ serving.
The subjects were divided into four groups as follows: control group, emulsified fatty food not administered; group 1, administration of 1 cup of emulsified fatty food 16 hours before endoscopy; group 2, administration of 1 cup of emulsified fatty food 4 hours before endoscopy; group 3, administration of 1 cup of emulsified fatty food 16 hours before endoscopy, followed by another 1 cup 4 hours before the procedure.

Two examinations were performed in this study. Patients with a diagnosis of gastric epithelial neoplasia (adenoma or cancer) underwent the initial endoscopic examination as an outpatient procedure. The second endoscopic examination was performed as an inpatient procedure after the patient had provided consent and been formally registered in the study. The patients then consumed the emulsified fatty food at the time assigned before the second examination. The second M-NBI examination was performed before ESD; as with the initial examination, images were stored for three sites on the oral side of the lesion.

Patients in the control group were not administered emulsified fatty food before the second examination. Patients in group 1 were administered 1 cup of emulsified fatty food 16 hours prior, patients in group 2 were administered 1 cup of emulsified fatty food 4 hours prior, and patients in group 3 were administered 1 cup of emulsified fatty food 16 hours prior, followed by a second cup 4 hours prior, for a total of 2 cups.

\section{Fat-loading test analysis}

The fat-loading test results were analyzed by two endoscopists who were experienced in gastric magnifying endoscopy and had at least three years' experience in M-NBI. The endoscopists had not been involved in the examination procedure in any way and were given no patient information. They assessed images randomly chosen from the first and second examinations. When their assessments of a particular image differed, the two endoscopists were asked to review the image and to make a decision after discussion.

The fat-loading test result (positive or negative) was determined by using the following WOS density ratings ( $\boldsymbol{O}$ Fig. $\mathbf{1}$ ): grade 0 , no evidence of WOS within the lesion; grade 1 , WOS observed in up to one-third of the lesion; grade 2, WOS observed in more than one-third but up to two-thirds of the lesion; grade 3, WOS observed in more than two-thirds of the lesion.

A result of the fat-loading test was positive when the WOS grade in the second endoscopic image was at least one level higher than the grade in the initial image; otherwise the result was negative.

\section{Primary end point}

The primary end point for this study was evidence of differences between groups in the fat-loading test results.

\section{Statistical analysis}

Statistical analysis was performed with SPSS software version 11.5J for Windows (SPSS Inc., Chicago, Illinois, USA). The chisquared test, Fisher's exact test, and one-way analysis of variance (ANOVA) were used for differences between groups in the rates of positive fat-loading test results. Differences between the average values for each group were considered statistically significant if the $P$ value was less than 0.05 . 

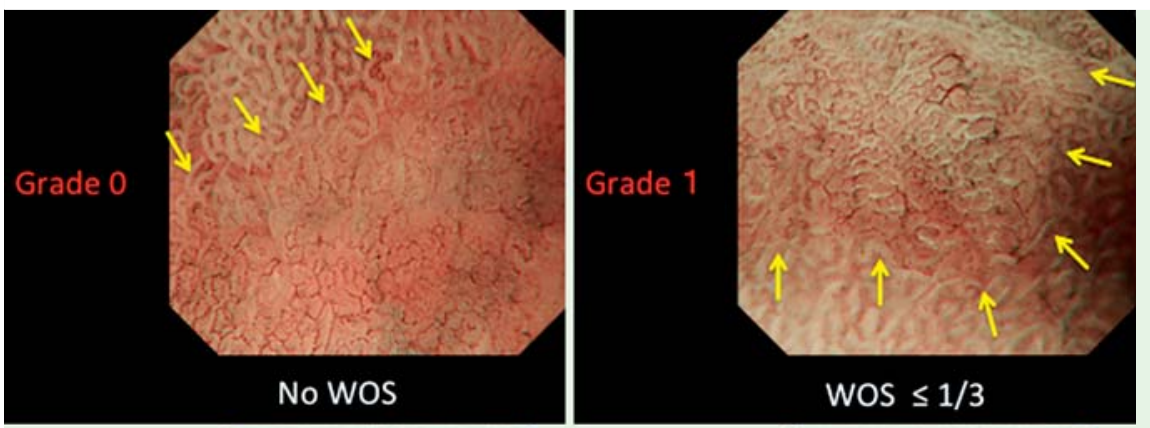

Fig. 1 White opaque substance (WOS) grading scale. WOS density is classified as follows (arrows indicate the demarcation line for gastric epithelial neoplasms): grade 0 , no evidence of WOS within the neoplasm; grade 1, WOS observed in up to onethird of the neoplasm; grade 2, WOS observed in more than one-third but in up to two-thirds of the neoplasm; grade 3, WOS observed in more than two-thirds of the neoplasm.
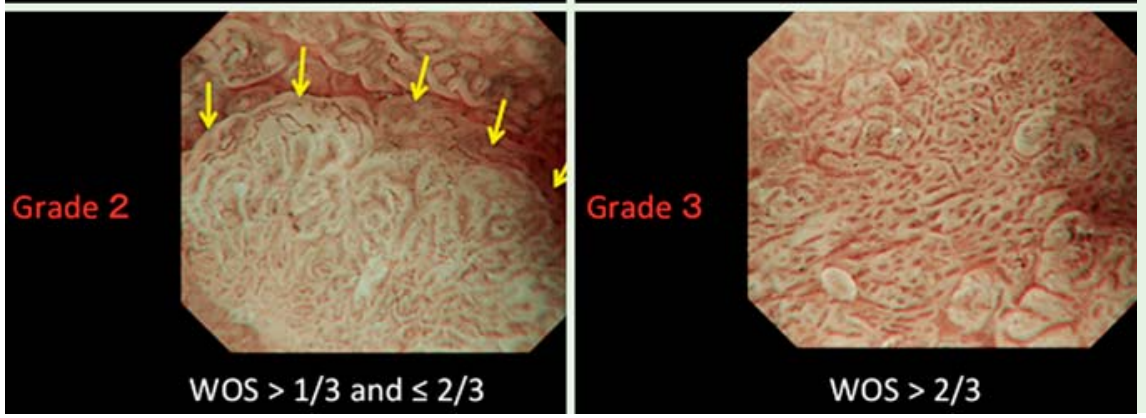

\section{Results}

$\nabla$

This study involved the examination of 92 lesions from January 2011 to November 2012. The lesions were divided into four groups of 23 each: a control group and groups 1, 2, and 3. Consent for participation was obtained from all patients. There were no withdrawals from the study.

- Table 1 and Table 2 show the demographic and clinical characteristics of the patients and lesions. No statistically significant differences were identified between groups with respect to average age, gender, lesion diameter, macroscopic type, or lesion site. - Table 3 shows the histopathologic findings. Again, there were no statistically significant differences between groups with respect to the histopathologic findings.

The rates of positive fat-loading test results for each group were as follows ( Fig. 2): control group, 9\% (2/23) [95\%CI 0-20.2\%]; group 1, 26\% (6/23) [95\%CI 10.7-41.5\%]; group 2, 48\% (11/23) [95\%CI 27.4-68.2\%]; group 3, 78\% (18/23) [95\%CI 61.4-95.1\%]. Positive results were significantly more likely in group 3 than in the control group $(P<0.001 ; \bullet$ Fig. 3$)$. In addition, the positive result rate was significantly higher in group 2 than in the control group $(P=0.022)$.

\begin{tabular}{|lllll|}
\hline & Control group $(\mathbf{n = 2 3 )}$ & Group $\mathbf{1}(\mathbf{n}=\mathbf{2 2})$ & Group $\mathbf{2}(\mathbf{n = 2 1 )}$ & Group 3 (n=23) \\
\hline Mean age (range),y & $67.7(51-83)$ & $76.3(61-87)$ & $71.8(36-87)$ & $70.1(58-83)$ \\
\hline Male-to-female ratio & $18: 5$ & $14: 8$ & $16: 5$ & $15: 8$ \\
\hline
\end{tabular}

\begin{tabular}{|lllll|}
\hline & Control group $(\mathbf{n = 2 3 )}$ & Group $\mathbf{1}(\mathbf{n = 2 3 )}$ & Group $\mathbf{2}(\mathbf{n = 2 3 )}$ & Group $\mathbf{3}(\mathbf{n}=\mathbf{2 3})$ \\
\hline Mean size of tumor (range), $\mathrm{mm}$ & 16.3 & 13.1 & 15.2 & 19.4 \\
& $(2-40)$ & $(3-25)$ & $(3-30)$ & $(3-42)$ \\
\hline $\begin{array}{l}\text { Morphologic type, } \mathrm{n}(\%)^{1} \\
\text { 0-I }\end{array}$ & $1(4)$ & $3(13)$ & $1(4)$ & $2(8)$ \\
0-Ila & $5(22)$ & $8(35)$ & $7(31)$ & $8(35)$ \\
0-IIb & $4(17)$ & $1(4)$ & $1(4)$ & $0(0)$ \\
0-Ilc & $13(57)$ & $11(48)$ & $14(61)$ & $13(57)$ \\
\hline Tumor location, $\mathrm{n}(\%)$ & & & \\
U & $6(26)$ & $5(22)$ & $6(26)$ & $4(17)$ \\
M & $8(35)$ & $9(39)$ & $8(35)$ & $7(31)$ \\
L & $9(39)$ & $9(39)$ & $9(39)$ & $12(52)$ \\
\hline
\end{tabular}

Table 1 Demographic characteristics of the patients in the analyzed groups.

Table 2 Characteristics of the lesions in the analyzed groups.

$\mathrm{U}$, upper; $\mathrm{M}$, middle; L, lower part of the stomach.

1 Paris classification.

Table 3 Histopathologic characteristics of lesions in the analyzed groups.

\begin{tabular}{|c|c|c|c|c|}
\hline Histologic type & Control group $(n=23)$ & Group $1(n=23)$ & Group $2(n=23)$ & Group $3(n=23)$ \\
\hline Low grade adenoma, $\mathrm{n}(\%)$ & $3(13)$ & $6(26)$ & $4(17)$ & $7(30)$ \\
\hline Adenocarcinoma of differentiated type, $n(\%)$ & $19(83)$ & $16(70)$ & $16(70)$ & $15(66)$ \\
\hline Adenocarcinoma of undifferentiated type, $n$ (\%) & $1(4)$ & $1(4)$ & $3(13)$ & $1(4)$ \\
\hline
\end{tabular}




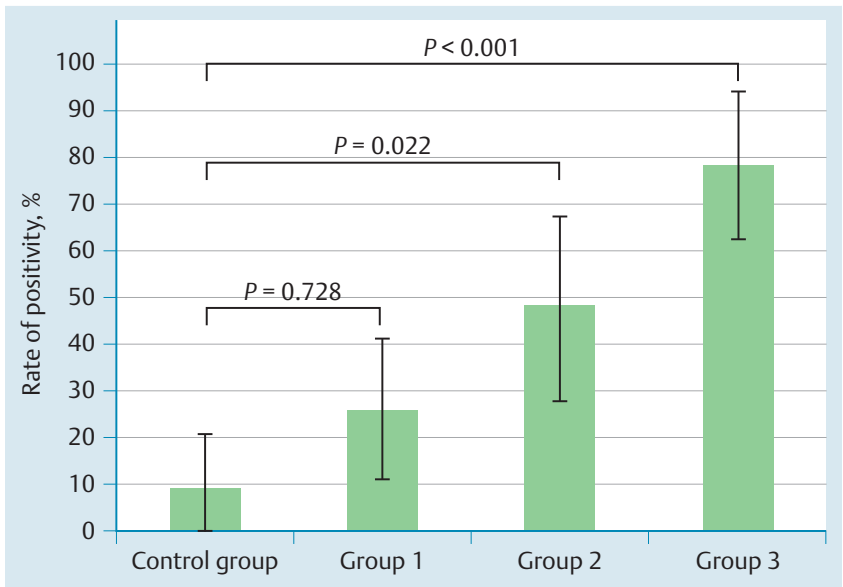

Fig. 2 Positive results in the fat-loading test. Control group, no emulsified lipid food loading; group 1, administration of 1 cup 16 hours before endoscopic examination; group 2, administration of 1 cup 4 hours before endoscopic examination; group 3, administration of 1 cup 16 hours before and 1 cup 4 hours before endoscopic examination (i. e., 2 loadings).

\section{Discussion}

The rates of positive results of the fat-loading test in group 2 (administered an emulsified fatty food 4 hours before examination) and group 3 (administered an emulsified fatty food 16 and 4 hours before examination) were significantly higher than the corresponding rates in the control group (patients not given the emulsified fatty food). We therefore concluded that the external administration of lipids caused a significant increase in WOS density in the gastric epithelial neoplasms. However, there was no statistically significant difference between the results for group 1 (emulsified fatty food administered 16 hours before ex- amination) and the control group, indicating that WOS density rises relatively quickly after ingestion (i.e., within 4 hours).

This study has shown that the external ingestion of lipids increases WOS density (i.e., the density of lipid droplets), suggesting that lipids are absorbed by gastric epithelial neoplasms. Rubin et al. reported that orally administered lipids (in the form of a liquid containing emulsified fats) were absorbed by the gastric mucosa associated with intestinal metaplasia [17]. To the best of our knowledge, however, ours is the first study to demonstrate conclusively that external lipids are absorbed by gastric epithelial neoplasms (cancer or adenoma) via the lumen.

The normal process for digestion and absorption of lipids is as follows: Orally ingested lipids are combined with lingual and gastric lipase in the stomach, where hydrolysis converts approximately $10 \%$ of triglycerides into glycerol and free fatty acids. Lipids passing through to the small intestine are emulsified by bile salts and lipid breakdown products, then dispersed through the aqueous solution in the form of minute lipid droplets. The digestion of lipid droplets is aided by pancreatic lipase, cholesterol esterase, phospholipase $\mathrm{A}_{2}$, and colipase. The end products of lipid digestion - monoglycerides, fatty acids, cholesterol, lysophospholipids, and free fatty acids - are solubilized within micelles, which are then dispersed to the brush borders of small-intestinal epithelial cells. The micelles are needed to pass through the brush border nondispersion layer. The lipids are released from the micelles at the lumen membrane and are dispersed through the cell in accordance with the concentration gradient [18-20].

Accordingly, lipid absorption requires the creation of emulsified micelles. The authors believe that the oral ingestion of emulsified fatty food is an effective way to promote lipid absorption in gastric epithelial neoplasms. We assumed that the absorption of emulsified fats in the stomach in this study might be due to the presence of lingual and gastric lipase, as well as pancreatic lipase and bile acids passing from the duodenum into the stomach. It
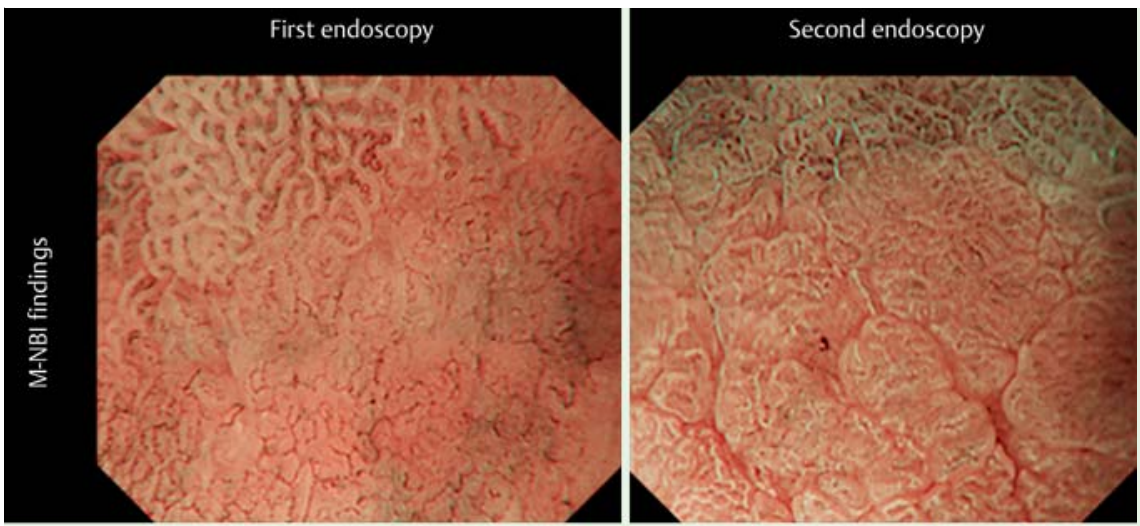

Fig. 3 Results of magnifying endoscopy with narrow-band imaging (M-NBI) before and after the fatloading test. An image from the first endoscopic examination is shown at the upper left, and an image from the second endoscopic examination is shown at the upper right. The microvascular architecture is traced with yellow lines at the bottom left, and the white opaque substance (WOS) is traced with white lines at the bottom right. It is obvious that the WOS density is increased after fat loading, obscuring the blood vessels.
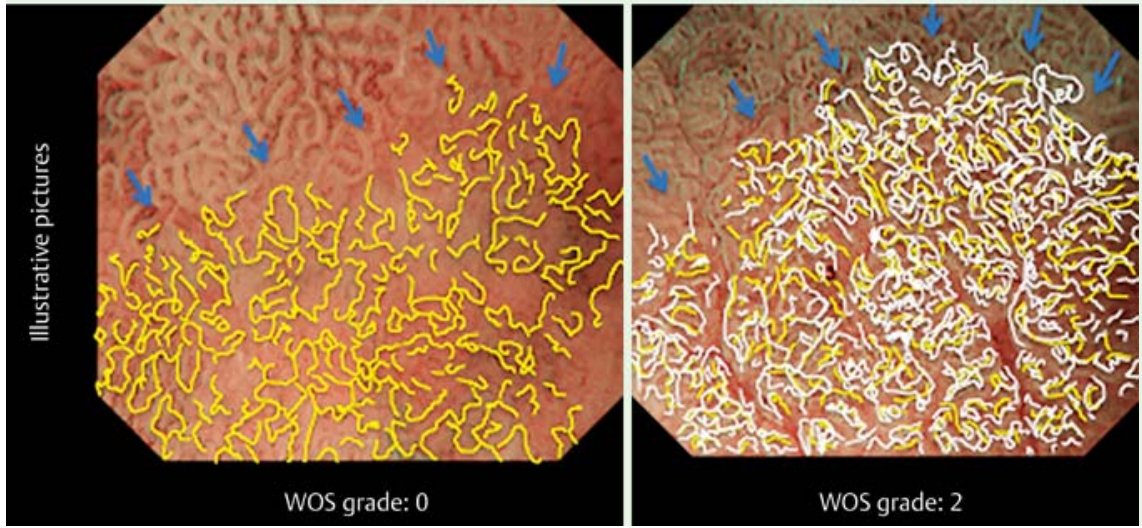
may also be that neoplastic change itself enables lipid absorption by the gastric epithelium [9]. This suggests the potential for developing a new technique to detect neoplasia based on the administration of food or formulations that are quickly absorbed by lesions, making them easier to identify. Such a technique could improve our ability to diagnose cancers accurately.

In this study, the sample size was not calculated because the study was a pilot study. The reason for this is that it was necessary to determine the sample size needed for a randomized, double-blinded trial, which is planned next. Specifically, this was a study to determine the optimal duration of the administration of fatty food and the number of patients needed. Limitations of this study are that it was not a blinded trial and did not have a randomized design, and that the number of patients needed based on the sample size was not calculated. Moreover, WOS is visible with both white-light imaging and NBI, but observation with optical magnification is needed [21]. Consequently, to obtain the present findings, an institution must currently have available the equipment for optical magnification endoscopy. These limitations will be addressed in a follow-up randomized double-blinded study (UMIN000010516), which is currently under way.

In conclusion, the authors have achieved a world first in showing that the oral ingestion of external lipids promotes the accumulation of intraepithelial lipid droplets in gastric epithelial neoplasms. The findings of this study can be used to develop a novel functional endoscopy technique based on the lipid absorption capacity of gastric tumors.

\section{Competing interests: None}

\section{Institutions}

${ }^{1}$ Department of Gastroenterology, Fukuoka University Chikushi Hospital, Fukuoka, Japan

${ }^{2}$ Department of Endoscopy, Fukuoka University Chikushi Hospital, Fukuoka, Japan

3 Faculty of Pharmaceutical Sciences, Fukuoka University, Fukuoka, Japan

${ }^{4}$ Department of Nutrition, Fukuoka University Chikushi Hospital, Fukuoka, Japan

${ }^{5}$ Department of Pathology, Fukuoka University Chikushi Hospital, Fukuoka, Japan

${ }^{6}$ Clinical Research Center, Wakayama Medical University, Wakayama, Japan

\section{References}

1 Yao K, Oishi T. Microgastroscopic findings of mucosal microvascular architecture as visualized by magnifying endoscopy. Dig Endosc 2001; 13: $27-S 33$

2 Yao K, Oishi T, Matsui $T$ et al. Novel magnified endoscopic findings of microvascular architecture in intramucosal gastric cancer. Gastrointest Endosc 2002; 56: 279-284

3 Yao K, Iwashita A, Tanabe $H$ et al. Novel zoom endoscopy technique for diagnosis of small flat gastric cancer: a prospective, blind study. Clin Gastroenterol Hepatol 2007; 5: 869-878
4 Yao K, Anagnostopoulos GK, Ragunath K et al. Magnifying endoscopy for diagnosing and delineating early gastric cancer. Endoscopy 2009; 41: $462-467$

5 Ezoe $Y$, Muto M, Uedo $N$ et al. Magnifying narrow band imaging is more accurate than conventional white-light imaging in diagnosis of gastric mucosal cancer. Gastroenterology 2011; 141: 2017-2025

6 Nagahama T, Yao K, Maki S et al. Usefulness of magnifying endoscopy with narrow-band imaging for determining the horizontal extent of early gastric cancer when there is an unclear margin by chromoendoscopy (with video). Gastrointest Endosc 2011; 72: 1259-1267

7 Maki S, Yao K, Nagahama T et al. Magnifying endoscopy with narrowband imaging is useful in the differential diagnosis between low-grade adenoma and early cancer of superficial elevated gastric lesions. Gastric Cancer 2013; 16: 140-146

8 Kanemitsu T, Yao K, Nagahama T et al. The vessels within epithelial circle (VEC) pattern as visualized by magnifying endoscopy with narrowband imaging (ME-NBI) is a useful marker for the diagnosis of papillary adenocarcinoma: a case-controlled study. Gastric Cancer 2014; 17: 469-477

9 Yao K, Doyama H, Gotoda T et al. Diagnostic performance and limitations of magnifying narrow-band imaging in screening endoscopy of early gastric cancer: a prospective multicenter feasibility study. Gastric Cancer 2014; 17: 669-679

10 Fujiwara S, Yao K, Nagahama $T$ et al. Can we accurately diagnose minute gastric cancers $(\leq 5 \mathrm{~mm})$ ? Chromoendoscopy (CE) vs magnifying endoscopy with narrow band imaging (M-NBI) Gastric Cancer 2014: Epub 2014 July 9

11 Yao K, Iwashita A, Tanabe $H$ et al. White opaque substance within superficial elevated gastric neoplasia as visualized by magnification endoscopy with narrow-band imaging: a new optical sign for differentiating between adenoma and carcinoma. Gastrointest Endosc 2008; 68: $574-580$

12 Yao K, Iwashita A, Nambu M et al. Nature of white opaque substance in gastric epithelial neoplasia as visualized by magnifying endoscopy with narrow-band imaging. Dig Endosc 2012; 24: 419-425

13 Ueo T, Yonemasu $H$, Yada $N$ et al. White opaque substance represents an intracytoplasmic accumulation of lipid droplets: immunohistochemical and immunoelectron microscopic investigation of 26 cases. Dig Endosc 2013; 25: 147-155

14 Oken MM, Creech RH et al. Toxicity and response criteria of the Eastern Cooperative Oncology Group. Am J Clin Oncol 1982; 5: 649-655

15 Schlemper RJ, Riddell RH, Kato $Y$ et al. The Vienna classification of gastrointestinal epithelial neoplasia. Gut 2000; 47: 251-255

16 Schlemper RJ, Kato Y, Stolte M. Diagnostic criteria for gastrointestina carcinoma in Japan and Western countries: proposal for a new classification system of gastrointestinal epithelial neoplasia. J Gastroenterol Hepatol 2000; 15: C52-C60

17 Rubin $W$, Ross $L L$, Jeffries $G H$ et al. Some physiologic properties of heterotopic intestinal epithelium. Its role in transporting lipid into the gastric mucosa. Lab Invest 1967; 16: 813-827

18 Imaizumi K, Sato M. Digestion, absorption and transport of lipids. Nippon Rinsho 2001; 59: 457-462

19 Costanzo LS. Physiology. 5th. edn. Philadelphia, PA: Elsevier; 2013: $320-383$

20 Johnson LR. Physiology of the gastrointestinal tract. New York, NY: Raven Press; 1981: 1147-1220

21 Yao K, Nagahama T, Iwashita A. "White opaque substance" and "light blue crest" within gastric flat tumors or intestinal metaplasia: same or different signs? Gastrointest Endosc 2009; 70: $402-403$ 